\title{
Effect of Femoral Head Necrosis Cystic Area on Femoral Head Collapse and Stress Distribution in Femoral Head
}

\section{Zhikun Zhuang}

Guangzhou University of Chinese Medicine

Tianye Lin

Guangzhou University of Chinese Medicine

\section{Wenting Song}

Guangzhou University of Chinese Medicine

Yuan Zhong

Guangzhou University of Chinese Medicine

\section{Peng Yang}

Guangzhou University of Chinese Medicine

\section{Pan Deng}

Guangzhou University of Chinese Medicine

\section{Fan Yang}

Guangzhou University of Chinese Medicine

Wei He

Guangzhou University of Chinese Medicine

Qiushi Wei ( $D$ weiqshi@163.com )

Guangzhou University of Chinese Medicine

\section{Research Article}

Keywords: Osteonecrosis of the femoral head, cystic area, collapse, mechanism, finite element

Posted Date: July 7th, 2021

DOI: https://doi.org/10.21203/rs.3.rs-663769/v1

License: (c) (i) This work is licensed under a Creative Commons Attribution 4.0 International License. Read Full License 


\section{Abstract}

Objective: Osteonecrosis of the femoral head (ONFH) is a common and difficult disease. The effect of cystic area on femoral head mechanics in patients with ONFH is unknown. The purpose of this study was to investigate the effect of cystic areas of osteonecrosis of the femoral head on stress distribution and disease progression in the femoral head.

Methods: A total of 85 patients (106 hips) diagnosed with ARCO stage II non-traumatic and non-surgical treatment of ONFH from February 2017 to November 2018 were retrospectively analyzed. All patients were followed up for an average of more than 2 years. According to whether the femoral head collapsed during the follow-up, they were divided into collapse group and non-collapse group. The age, gender, etiology, height, weight, BMI, JIC classification, presence of cystic areas and diameter of cystic areas were compared between the two groups. In addition, five spherical cystic areas of different diameters of 0,5 , 10,15 , and $20 \mathrm{~mm}$ were constructed to simulate the hip joint load of a person during standing by finite element methods, and the maximum stress, mean stress, and maximum stress values in the necrotic area of the femoral head without cystic areas and in the area $1 \mathrm{~mm}$ around the cystic areas with different diameters were observed and analyzed.

Results: All 85 patients (106 hips) completed the follow-up, with an average age of $37.4 \pm 8.12$ years. The mean follow-up time was $2.8 \pm 0.6$ years. Forty-five patients ( 57 hips) with ONFH who had femoral head collapse were included in the collapse group, and the remaining 40 patients (49 hips) were included in the non-collapse group. There was significant difference in JIC classification between the two groups $(P<$ 0.05), most of which were $C 2$ type in the collapse group and $B$ type in the non-collapse group. There was a difference between the two groups in whether cystic areas appeared in the femoral head, with $49.1 \%$ in the collapse group showing cystic areas, which was significantly higher than that in the non-collapse group $(18.4 \%)(P<0.05)$. In addition, the diameter of the cystic areas was significantly larger in the collapsed group than in the non-collapsed group $(P<0.05)$. The maximum von Mises stress value and mean von Mises stress value around the cortical bone, necrotic area and around the cystic area of the femoral head increased with the increase of the cystic diameter. Furthermore, linear regression analysis showed a linear positive correlation between the maximum stress, mean stress in the necrotic area in the femoral head, and the maximum stress value in the $1 \mathrm{~mm}$ area around the cystic area and the diameter of the cystic area.

Conclusion: The maximum stress and average stress of necrotic area can be increased in cystic area, and the increase of stress in cystic area is more obvious. Stress concentration areas can be generated around the cystic areas. The presence and increased diameter of the cystic areas accelerates the collapse of the ONFH femoral head.

\section{Introduction}


Osteonecrosis of the femoral head (ONFH) is a disease in which the femoral head is interrupted or damaged, causing the death of osteocytes and bone marrow components, and subsequent repair leads to collapse of the femoral head, and the specific pathogenesis has not been fully clarified ${ }^{[1,2]}$. Changes such as crescent sign, cystic areas, and articular surface collapse occur radiologically during the progression of osteonecrosis of the femoral head ${ }^{[3]}$. Among them, collapse is an important marker of the progression of osteonecrosis of the femoral head. Collapse of the articular surface of the femoral head can be secondary to osteoarthritis of the hip, leading to severe hip pain and dysfunction and eventually necessitating joint replacement ${ }^{[4-5]}$. Therefore, it is important to identify the factors inducing collapse in the early stage of the disease and take appropriate interventions in time for the prognosis of patients.

In recent years, it has been shown that cystic areas can increase the risk of femoral head collapse by disrupting the mechanical stability of the femoral head, thereby accelerating the progression of the disease and affecting the prognosis of patients ${ }^{[6]}$.The resorption of bone structure disappears and is replaced by tissue as a typical manifestation of ARCO stage II, which shows a radiolucent area with reduced density on X-ray ${ }^{[7]}$.Pathological studies suggest that the cavity in the cystic area is mainly filled by some fibrous granulation like tissue ${ }^{[8]}$.Femoral head necrosis with cystic areas is more likely to present with microfractures and collapse, suggesting instability of the femoral head structure, so it is believed that cystic areas of femoral head necrosis play an important role in aggravating the process of femoral head collapse ${ }^{[9]}$.At present, there are two hypotheses about the collapse mechanism of osteonecrosis of the femoral head: one is that osteoclasts are active resulting in reduced bone strength, which reduces their ability to bear the articular surface ${ }^{[10]}$.The other is that the repaired area is thickened, which produces stress concentration at the junction with the necrotic area ${ }^{[11]}$.The replacement of the bone in the cystic area by fibrous granulation tissue also forms a cavity inside the femoral head, and the cystic area is mainly located in the weight-bearing area of the femoral head ${ }^{[6]}$.The effect of cystic area on femoral head mechanics in patients with ONFH is unknown. We speculated that the cystic area would reduce the weight-bearing ability of the femoral head and destroy the normal mechanical stability of the femoral head, thereby accelerating the collapse of the femoral head.In this study, the incidence and diameter of femoral head collapse and non-collapse cystic degeneration in ONFH patients were retrospectively analyzed, and simulated the stress distribution in the necrotic area and $1 \mathrm{~mm}$ area around the cystic area when there were different diameter cystic areas by finite element method. The aim of this study is to investigate the effect of cystic area on the stress distribution in the femoral head and its role in the progression of the disease and explore the formation mechanism of cystic area of osteonecrosis of the femoral head and its guiding role in the treatment of osteonecrosis.

\section{Methods}

\section{Study subjects}

Patients with non-traumatic, non-surgical ONFH admitted to the First Affiliated Hospital of Guangzhou University of Traditional Chinese Medicine from February 2017 to November 2018 were analyzed. 
Inclusion criteria were: (i) complete imaging data, no femoral head collapse at the first visit (ARCO stage II). ii) Age 20-55 years. (iii) No history of hip trauma or surgery. Exclusion criteria: (i) incomplete imaging data during follow-up; (ii) patients with cardiovascular and cerebrovascular diseases or rheumatoid arthritis. The age, gender, etiology, and follow-up time of the patients were recorded, and imaging data such as X-ray and CT were collected. All patients were followed up for an average of more than 2 years, and the presence of $>2 \mathrm{~mm}$ on the surface of the femoral head was defined as collapse ${ }^{[12]}$. According to whether the femoral head collapsed during the follow-up, the patients are divided into collapse group and non-collapse group.This study was approved by the ethical review board of The First Affiliated Hospital of Guangzhou University of Chinese Medicine(No: Y®2019ه118).

\section{Establishment of 3D models}

A 30-year-old healthy woman was excluded from our analysis due to the history of hip and systemic diseases. DICOM data obtained from computerized tomography(CT) scans were CT data is collected to construct a hip joint model through Mimics 16.0system,Geomagic-Studio 11 system and Solidworks 2014 software. The Japanese Investigation Committee (JIC) type C1 ONFH was used as a model reference to establish a necrotic zone model. A circle a was drawn around the center of the femoral head using the Solidworks software. Finally, the JIC type $\mathrm{C} 1 \mathrm{ONFH}$ model including the necrotic area was established. Three-dimensional models of ONFH with 0, 5, 10, 15 and $20 \mathrm{~mm}$ diameter cystic areas were constructed in the anterolateral weight-bearing area of the femoral head.

\section{Material properties and boundary conditions}

The Poisson's ratio of cystic area was 0.49 , and the elastic modulus was $1 \mathrm{MPa}{ }^{[13]}$.As with details reported by Grecu D ${ }^{[14]}$, cortical bone, cancellous bone and cartilage were defined as isotropic, continuous and uniform elastic materials. The Poisson's ratio and elastic modulus of cortical bone, cancellous bone, necrotic tissue and cartilage are shown in Table 1.The 6 degrees of freedom at the sacroiliac joint and pubic symphysis were zero. Based on the settings reported by Brown et al. ${ }^{[15]}$, the joint force was 1.6 times the body weight and loaded on the rigid body of the distal femur (Fig. 1). Friction was defined as the tangential force between the surfaces of the femoral cartilage and the acetabular cartilage, with a coefficient of friction of 0.2 . The binding relation was defined as relationship of other model parts. The results of convergence tests for the element size showed that errors were below $10 \%$. 
Table 1

Assignment of material properties

\begin{tabular}{|lll|}
\hline Material & Elastic Modulus(MPa) & Poisson's ratio \\
\hline Cortical bone & 15100 & 0.3 \\
\hline Cancellous bone & 4457 & 0.22 \\
\hline Cartilage & 10.5 & 0.45 \\
\hline Necrotic tissue & 124.6 & 0.152 \\
\hline Cystic lesion & 1 & 0.49 \\
\hline
\end{tabular}

\section{Observation indicators}

In terms of clinical studies, age, gender, etiology, height, weight, BMI, JIC classification, presence of cystic areas and diameter of cystic areas were compared between the collapse and non-collapse groups. The bone structure disappeared, and the main manifestation was hypodense radiolucent area on CT images, according to which it was determined whether the affected hip contained cystic area. On the CT image of femoral head, take the middle position of coronal plane of cystic area, measure the size of their mutually perpendicular long diameter and transverse diameter, and take the average value of long diameter and transverse diameter as the diameter of cystic area. In addition, record the maximum von Mises stress and the average von Mises stress on the head surface (take the corresponding 10-point von Mises stress on the head surface of different models and calculate its average). Furthermore, the maximum von Mises stress and mean von Mises stress in the necrotic area were measured to analyze the relationship between the two and the diameter of the cystic area. Finally, the maximum von Mises stress in the $1 \mathrm{~mm}$ area around the cystic area was measured: the effect of the cystic area on the stress distribution in the surrounding area was analyzed, and a spherical shell with a radial extension of $1 \mathrm{~mm}$ around the simulated cystic area was used as the area to measure the von Mises stress ${ }^{[16]}$. The relationship between the maximum stress in this region and the diameter of the cystic region was analyzed.

\section{Statistical Methods}

SPSS 24.0 statistical software was used for analysis. Measurement data were expressed as mean \pm standard deviation, independent sample t-test was used for comparison; enumeration data were expressed as rate, and $\chi^{2}$ test or rank sum test was used for comparison. Simple linear regression analysis was used to analyze the relationship between the diameter of the bone resorption area and the maximum von Mises stress in the cortical bone of the femoral head, the maximum von Mises stress in the necrotic area, and the maximum von Mises stress in the $1 \mathrm{~mm}$ area around the cysts. $\mathrm{P}<0.05$ was considered statistically significant. 


\section{Results}

\section{General information}

A total of 85 patients (44 males and 41 females) with 106 hips, including 21 patients with ONFH, were enrolled in this study. The age ranged from 21 to 60 years with a mean age of $37.4 \pm 8.12$ years. The follow-up time was $2-4$ years, with an average of $2.8 \pm 0.6$ years. Forty-five patients ( 57 hips) with ONFH who had femoral head collapse were included in the collapse group, and the remaining 40 patients (49 hips) were included in the non-collapse group. There was significant difference in JIC classification between the two groups $(P<0.05)$, most of which were $C 2$ type in the collapse group and $B$ type in the non-collapse group. There was a difference between the two groups in whether there was a cystic area in the femoral head, with $49.1 \%$ in the collapse group showing a cystic area, which was significantly higher than that in the non-collapse group $(18.4 \%)$, and the difference was statistically significant $(P<0.05)$. In addition, the diameter of the cystic areas was significantly larger in the collapsed group than in the noncollapsed group $(P<0.05)$. There was no significant difference in age, gender, height, weight, $B M I$, and etiology between the two groups $(P>0.05)$ (Table 2$)$. 
Table 2

Comparison of general data between the two groups

\begin{tabular}{|c|c|c|c|c|}
\hline Parameters & $\begin{array}{l}\text { Collapse group } \\
(\mathrm{n}=45(57 \text { hips }))\end{array}$ & $\begin{array}{l}\text { Non-collapse group } \\
\text { ( } n=40(49 \text { hips)) }\end{array}$ & $t / x^{2}$ & $P$-value \\
\hline Age (years) & $37.0 \pm 11.36$ & $37.9 \pm 6.70$ & -0.496 & 0.621 \\
\hline \multicolumn{5}{|l|}{ Gender,n (\%) } \\
\hline Male & $23(51.1 \%)$ & $21(52.5 \%)$ & \multirow[t]{2}{*}{0.016} & \multirow[t]{2}{*}{0.898} \\
\hline Female & $22(48.9 \%)$ & $19(47.5 \%)$ & & \\
\hline Height(cm) & $164.6 \pm 7.43$ & $164.7 \pm 7.26$ & -0.047 & 0.962 \\
\hline Weight(kg) & $65.7 \pm 13.6$ & $63.9 \pm 11.6$ & 0.731 & 0.466 \\
\hline $\mathrm{BMI}\left(\mathrm{kg} / \mathrm{m}^{2}\right)$ & $23.4 \pm 3.68$ & $23.8 \pm 3.97$ & 0.501 & 0.617 \\
\hline \multicolumn{5}{|l|}{ Etiology,n(\%) } \\
\hline Steroid & $16(35.6 \%)$ & $14(35.0 \%)$ & \multirow[t]{3}{*}{0.008} & \multirow[t]{3}{*}{0.996} \\
\hline Alcoholic & $17(37.8 \%)$ & $15(37.5 \%)$ & & \\
\hline Idiopathic & $12(26.7 \%)$ & $11(27.5 \%)$ & & \\
\hline \multicolumn{5}{|l|}{ JIC type,n(\%) } \\
\hline Type-A & $0(0 \%)$ & $2(5.0 \%)$ & \multirow[t]{4}{*}{37.25} & \multirow[t]{4}{*}{0.000} \\
\hline Type-B & $6(13.3 \%)$ & $28(70.0 \%)$ & & \\
\hline Type-C1 & $18(40.0 \%)$ & $9(22.5 \%)$ & & \\
\hline Type-C2 & $21(46.7 \%)$ & $1(2.5 \%)$ & & \\
\hline \multicolumn{5}{|l|}{ Cystic change area,n(\%) } \\
\hline Yes & $28(49.1 \%)$ & $9(18.4 \%)$ & \multirow[t]{2}{*}{10.969} & \multirow[t]{2}{*}{0.0012} \\
\hline No & $29(50.9 \%)$ & $40(81.6 \%)$ & & \\
\hline Cystic change area diameter $(\mathrm{mm})$ & $15.8 \pm 5.33$ & $6.3 \pm 2.94$ & 10.995 & 0.000 \\
\hline
\end{tabular}

\section{Von Mises Stress Values for Different Capsule Diameter Models}

The maximum and average stress values of cortical bone in the weight-bearing area of femoral head were the smallest in the $0 \mathrm{~mm}$ diameter cystic area model. The mean stress value of cortical bone in the weight-bearing area of the femoral head increased with the increase of the diameter of the resorption area, and the difference was statistically significant $(P<0.05)$. Similarly, the $0 \mathrm{~mm}$ model had the smallest mean and maximum stresses in the necrotic zone. The mean stress value of the necrotic area 
increased with the increase of the diameter of the absorption area, and there was no statistically significant difference between the $15 \mathrm{~mm}$ model and the $20 \mathrm{~mm}$ model, and the other differences were statistically significant $(P<0.05)$. Furthermore, the maximum stress and mean stress around the cystic area increased with the increase of the diameter of the absorption area, and there was a significant difference in the mean stress around the cystic area $(P<0.05)$ (Table 3 Fig. 2$)$.

Table 3

Von Mises Stress Values (MPa) for Different Capsule Diameter Models

\begin{tabular}{|c|c|c|c|c|c|c|}
\hline Grouping & $\begin{array}{l}\text { Maximum } \\
\text { stress of } \\
\text { cortical } \\
\text { bone }\end{array}$ & $\begin{array}{l}\text { Average } \\
\text { stress of } \\
\text { cortical } \\
\text { bone }\end{array}$ & $\begin{array}{l}\text { Maximum } \\
\text { stress of } \\
\text { necrotic } \\
\text { area }\end{array}$ & $\begin{array}{l}\text { Average } \\
\text { stress of } \\
\text { necrotic } \\
\text { area }\end{array}$ & $\begin{array}{l}\text { Maximum } \\
\text { stress of } \\
\text { cystic area }\end{array}$ & $\begin{array}{l}\text { Average } \\
\text { stress of } \\
\text { cystic area }\end{array}$ \\
\hline $0 \mathrm{~mm}$ & 21 & $\begin{array}{l}20.19 \pm \\
1.01\end{array}$ & 3.11 & $2.85 \pm 0.33$ & - & - \\
\hline $5 \mathrm{~mm}$ & 23.5 & $\begin{array}{l}22.45 \pm \\
1.13^{*}\end{array}$ & 3.55 & $\begin{array}{l}3.43 \pm \\
0.09^{*}\end{array}$ & 4.03 & $3.72 \pm 0.36$ \\
\hline $10 \mathrm{~mm}$ & 25.4 & $\begin{array}{l}24.0 \pm \\
1.52^{\star}\end{array}$ & 4.95 & $\begin{array}{l}4.75 \pm \\
0.17^{\star \#}\end{array}$ & 5.59 & $\begin{array}{l}5.23 \pm \\
0.38^{\#}\end{array}$ \\
\hline $15 \mathrm{~mm}$ & 27.3 & $\begin{array}{l}26.3 \pm \\
1.07 * \# \boldsymbol{\Delta}\end{array}$ & 7.92 & $\begin{array}{l}7.40 \pm \\
0.94^{\star \# \Delta}\end{array}$ & 8.29 & $\begin{array}{l}8.01 \pm \\
0.46^{\# \Delta}\end{array}$ \\
\hline $20 \mathrm{~mm}$ & 30.5 & $\begin{array}{l}29.1 \pm \\
1.10 * \# \Delta \square\end{array}$ & 9.29 & $\begin{array}{l}8.08 \pm \\
0.90 * \# \Delta\end{array}$ & 9.37 & $\begin{array}{l}9.19 \pm \\
0.13^{\# \Delta \rrbracket}\end{array}$ \\
\hline F-value & - & 84.35 & - & 147.74 & - & 1416.9 \\
\hline$P$-value & - & $<0.001$ & - & $<0.001$ & - & $<0.001$ \\
\hline \multicolumn{7}{|c|}{$\begin{array}{l}\text { *: Compared with the } 0 \mathrm{~mm} \text { model, the difference was statistically significant.\#:Compared with the } 5 \\
\text { mm model, the difference was statistically significant.A:Compared with the } 10 \mathrm{~mm} \text { model, the } \\
\text { difference was statistically significant. } ₫: \text { Compared with the } 15 \mathrm{~mm} \text { model, the difference was } \\
\text { statistically significant. }\end{array}$} \\
\hline
\end{tabular}

\section{Relationship between the von Mises Stress of Model and Cystic Area Diameter}

The maximum von Mises stresses in the femoral head cortex were 21, 23.5, 25.4, 27.3, and 30.5 in the 0 , $5,10,15$, and $20 \mathrm{~mm}$ FE models of the cystic region diameter, respectively. The relationship between the diameter of the cystic area and the maximum von Mises stress of the femoral head cortical bone was fitted by a linear regression curve, and the results showed that the diameter of the cystic area was linearly positively correlated with the maximum von Mises stress of the femoral head cortical bone $\left(R^{2}=0.9903\right.$, $F=307.0, P=0.0004)$, and the fitted regression equation was: $Y=0.4560 \star X+20.98$. In addition, the maximum von Mises stresses in the necrotic zone were 3.11, 3.55, 4.95, 7.92, and 9.29 in the finite element models with $0,5,10,15$, and $20 \mathrm{~mm}$ capsule diameter, respectively. The relationship between the diameter of the cystic area and the maximum von Mises stress in the necrotic area was fitted by a linear 
regression curve, and the results showed that the diameter of the cystic area was linearly positively correlated with the maximum von Mises stress in the necrotic area $\left(R^{2}=0.9427, F=49.40, P=0.0059\right)$, and the fitted regression equation was: $Y=0.3346 * X+2.418$ (Fig. 2).

\section{Relationship between von Mises stress around the cystic area and the diameter of the cystic area}

The maximum von Mises stresses around the cystic area were 4.03, 5.59, 8.29, and 9.37 in the 5, 10, 15, and $20 \mathrm{~mm}$ diameter finite element models of the cystic area, respectively. The relationship between the diameter of the cystic area and the maximum von Mises stress around the cystic area was fitted by linear regression curve. The results showed that the diameter of the cystic area was linearly positively correlated with the maximum von Mises stress of the cortical bone of the femoral head $\left(R^{2}=0.9756, F=79.92, P=\right.$ 0.0123), and the fitted regression equation was: $Y=0.4560 * X+20.98$ (Fig. 2). The finite element stress distribution clouds show that there is a stress concentration area above the cystic region, and this region range gradually increases with the increase of the diameter of the cystic region (Fig. 3).

\section{Typical case}

Typical case 1: Male, 36 years, with left hip pain for more than 7 months, diagnosed as steroid-induced osteonecrosis of the femoral head. This is an ONFH patient with ARCO II and JIC type B.CT showed no obvious cystic change area. After 6 months of non-surgical hip preservation treatment, the area of necrosis was reduced and the joint space was acceptable. After 1.5 years of non-surgical hip preservation treatment, the femoral head did not collapse significantly, and the density of the necrotic area increased(Fig. 4).

Typical case 2:Male, 45 years, with left hip pain for more than 9 months, diagnosed as steroid-induced osteonecrosis of the femoral head. graded as JIC type B.CT showed obvious cystic change area. After 1 year of non-surgical hip-preserving treatment, the femoral head collapsed and the patient's pain became a little worse. After 2 years of non-surgical hip preservation treatment, the femoral head has become slightly flat(Fig. 5).

\section{Discussion}

The cystic area is a cavity formed in the femoral head, and the cystic area is mainly located in the weightbearing area of the femoral head. However, the effect of cystic areas on femoral head mechanics in patients with ONFH is unknown. Therefore, we carried out a clinical retrospective study with finite element analysis. Our clinical study found cystic areas in $49.1 \%$ of patients in the collapse group, which was significantly higher than that in the non-collapse group (18.4\%). In addition, the diameter of the cystic areas significantly larger in the collapsed group than in the non-collapsed group. The finite element results shown that the maximum stress and average stress in the necrotic area increase gradually with the increase of the diameter of the cystic area, and there was a stress concentration area around the cystic area, and the maximum stress around it also increased with the increase of the diameter of the cystic area. 
The prognosis of osteonecrosis of the femoral head mainly depends on whether the articular surface is collapsed ${ }^{[17,18]}$. The disappearance of bone structure inside the cystic area can further reduce the weight-bearing ability of the femoral head and increase the risk of femoral head collapse, thereby accelerating disease progression ${ }^{[11]}$. The location of the lesion in the necrotic area is considered to be an important factor in collapse. One study found that the prognosis is often very poor when the necrotic area involves the lateral weight-bearing area ${ }^{[19]}$. Kubo et al. ${ }^{[20]}$ found that femoral head necrosis had a high collapse rate even when the necrotic focus involved more anterior regions, even when it was medial. The cystic area is mainly located in the anterolateral aspect of the femoral head, which may further reduce the mechanical strength and weight-bearing capacity of the femoral head and accelerate collapse. The sclerotic zone can delay or prevent collapse of the femoral head by providing mechanical support to the femoral head ${ }^{[21]}$. However, most of the cystic areas were connected to the sclerotic zone, which destroyed the integrity of the sclerotic margin, thereby reducing the protection of necrotic tissue and further increasing the risk of femoral head collapse ${ }^{[22]}$. The researchers analyzed the location characteristics of the cystic areas in the $\mathrm{CT}$ images and found that the cystic areas were mainly located in the intermediate and lateral columns of the femoral head. They concluded that this compromises the integrity of the principal compressive stress in the femoral head, damages the normal stress transfer pathway in the femoral head, increases the average stress in the femoral head, and thus accelerates the collapse of the femoral head ${ }^{[6]}$. In this study, we found that patients with ONFH had an increased risk of collapse when cystic areas appeared in the femoral head. In addition, the diameter of cystic areas was larger in the collapsed group than in the non-collapsed group.

The data of CT tomography yielded that the cystic areas were mainly located on the anterior, intermediate, lateral columns of the femoral head and near the sclerotic zone ${ }^{[6]}$.Therefore, it is speculated that the formation of cystic areas may be due to stress-induced bone resorption. At stress concentrations in the interior of the femoral head, bone microfractures occur when the stress on the bone exceeds its maximum bearing strength ${ }^{[23]}$. Constant stress action or increased weight-bearing can allow this process to occur repeatedly, with subsequent resorption of fracture fragments by osteoclasts and replacement by fibrous granulation tissue ${ }^{[24]}$. It has been reported in the literature that during the occurrence and development of osteoarthritic cystic areas, pathological changes caused by increased stress can enhance macrophage responses, and macrophages promote osteoclast resorption in the subchondral bone around osteoarthritic cystic areas through macrophage-osteoclast differentiation and play a role in the osteolysis process of increased cystic areas. Because similar tissue components exist between osteoarthritis and cystic areas of osteonecrosis of the femoral head, biological factors may also help to promote the maintenance and expansion of cystic areas in osteonecrosis of the femoral head ${ }^{[25]}$. However, at present we have not further tested the hypothesis. In this study, according to the results of previous studies, the main part of the simulated cystic area was designed to be located in the anterolateral region of the femoral head, which was in line with the actual distribution characteristics of the cystic area in clinical practice. Previous studies have confirmed that the reduction of bone structure strength in the necrotic area and the appearance of stress concentration areas in the femoral head are the 
main causes of collapse ${ }^{[26]}$. The results of our finite element study suggest that the formation of the cystic area increases the maximum stress and mean stress of the necrotic area, and there is a stress concentration area around the cystic area, thus increasing the risk of bone fracture. Although the optimal treatment strategy for osteonecrosis of the femoral head remains controversial, it is generally believed

that aggressive hip preservation treatment before collapse can improve the prognosis ${ }^{[27,28]}$.Considering that cystic areas increase the risk of collapse, we recommend that patients with cystic areas should be carefully evaluated and closely followed up in order to take timely intervention measures when the disease progresses.

The innovation of this study was to investigate the relationship between cystic areas and femoral head collapse, which was verified by finite element analysis. However, there are still some limitations in this study. First, the clinical part of this study is a single-center retrospective analysis study with a limited number of cases, which may have selection bias. Secondly, our study only investigated the effect of areas with a high incidence of cystic areas on the mechanical properties of the femoral head, and the observation of the morphological characteristics of cystic areas also only set up an ideal model of spherical cystic areas. In fact, the shape of cystic areas is irregular, some cystic areas are surrounded by sclerotic areas, and the cystic areas located in the anterolateral side may also destroy the subchondral bone plate, so it is necessary to further analyze the effect of other areas and cystic areas with different morphological characteristics on the progression of osteonecrosis of the femoral head.

\section{Conclusion}

The maximum stress and average stress of necrotic area can be increased in cystic area, and the increase of stress in cystic area is more obvious. Stress concentration areas can be generated around the cystic areas. The presence and increased diameter of the cystic areas accelerates the collapse of the ONFH femoral head.

\section{Abbreviations}

ONFH: Osteonecrosis of the Femoral Head. CT: Computed tomography. DICOM: Digital imaging and communications in medicine. BMI: Body Mass Index. JIC: Japanese Investigation Committee. ARCO: Association Research Circulation Osseous.

\section{Declarations}

\section{Acknowledgement}

Grateful acknowledgement is made to my supervisor Professor He Wei who gave me considerable help by means of suggestion, comments and criticism. In addition, I deeply appreciate the contribution to this thesis made in various ways by my friends and classmates.

\section{Authors' contributions}

Page $11 / 19$ 
Z.Z.K was responsible for the design and implementation of the study presented. L.T.Y, Y.P., S.W.T,Y.F, and Z.Y. conducted the finite element analysis and were responsible for the acquisition of the data. Z.Z.K prepared the initial draft of the manuscript. H.W. and W.Q.S. gave critical feedback during the study and critically revised the submitted manuscript for important intellectual content. All authors have read and approved the final manuscript to be submitted.

\section{Funding}

Publication charges for this article were funded by Natural Science Foundation of China $(81873327,81904226)$.

\section{Availability of data and materials}

All the data will be available upon motivated request to the corresponding author of the present paper.

\section{Ethical approval}

This study was conducted in agreement with the Declaration of Helsinki and its later amendments or comparable ethical standards and had been approved by the ethics board of The First Affiliated Hospital of Guangzhou University of Chinese Medicine (No: Y®2019囚118).

\section{Consent for publication}

Not applicable

\section{Competing interests}

The authors declare that they have no competing interests.

\section{References}

1. Hines Jeremy T, Jo Woo Lam, Cui Quanjun et al. Osteonecrosis of the Femoral Head: an Updated Review of ARCO on Pathogenesis, Staging and Treatment.[J] .J Korean Med Sci, 2021, 36: e177.

2. Wang Peixu, Liu Xingyu, Xu Jia et al. Deep learning for diagnosing osteonecrosis of the femoral head based on magnetic resonance imaging.[J]. Comput Methods Programs Biomed, 2021, 208: 106229.

3. Serong Sebastian, Haubold Johannes, Theysohn Jens et al. Arthroscopic assessment of concomitant intraarticular pathologies in patients with osteonecrosis of the femoral head.[J] .J Hip Preserv Surg, 2020, 7: 458-465.

4. Richard Marc J, DiPrinzio Eliseo V, Lorenzana Daniel J et al. Outcomes of free vascularized fibular graft for post-traumatic osteonecrosis of the femoral head.[J] .Injury, 2021, undefined: undefined.

5. Liu Ming, Zhao Gan, Wei Biao-Fang, Attenuated serum vasoactive intestinal peptide concentrations are correlated with disease severity of non-traumatic osteonecrosis of femoral head.[J] .J Orthop Surg Res, 2021, 16: 325. 
6. Gao Fuqiang, Han Jun, He Zike et al. Radiological analysis of cystic lesion in osteonecrosis of the femoral head.[J] .Int Orthop, 2018, 42: 1615-1621.

7. Sugano Nobuhiko, Atsumi Takashi, Ohzono Kenji et al. The 2001 revised criteria for diagnosis, classification, and staging of idiopathic osteonecrosis of the femoral head.[J] .J Orthop Sci, 2002, 7: 601-5.

8. Resnick D, Niwayama G, Coutts RD. Subchondral cysts (geodes) in arthritic disorders: pathologic and radiographic appearance of the hip joint[J]. AJR Am J Roentgenol, 1977, 128(5): 799-806.

9. Baba Shoji, Motomura Goro, Ikemura Satoshi et al. Quantitative evaluation of bone-resorptive lesion volume in osteonecrosis of the femoral head using micro-computed tomography.[J] .Joint Bone Spine, 2020, 87: 75-80.

10. Li Guomin, Li Bing, Li Bo et al. The role of biomechanical forces and MALAT1/miR-329-5p/PRIP signalling on glucocorticoid-induced osteonecrosis of the femoral head.[J] .J Cell Mol Med, 2021, 25: 5164-5176.

11. Hamada $H$, Takao M, Sakai T, et al. Subchondral fracture begins from the bone resoq)tion area in osteonecrosis of the femoral head: a micro - computerised tomography study[J]. Int Orthop, 2018,42(7): 1479-1484.

12. Yoon Byung-Ho,Mont Michael A, Koo Kyung-Hoi et al. The 2019 Revised Version of Association Research Circulation Osseous Staging System of Osteonecrosis of the Femoral Head.[J] .J Arthroplasty, 2020, 35: 933-940.

13. Grecu D, Pucalev I, Negru M, Tarnita DN, lonovici N, Dita R. Numerical simulations of the 3D virtual model of the human hip joint, using finite element method. Rom J Morphol Embryol 2010;51: 151-5.

14. Frazer Lance L, Santschi Elizabeth M, Fischer Kenneth J, The impact of subchondral bone cysts on local bone stresses in the medial femoral condyle of the equine stifle joint.[J] .Med Eng Phys, 2017, 48: 158-167.

15. Sverdlova Nina S, Witzel Ulrich, Principles of determination and verification of muscle forces in the human musculoskeletal system: Muscle forces to minimise bending stress.[J] .J Biomech, 2010, 43: 387-96.

16. McErlain David D, Milner Jaques S, Ivanov Todor $G$ et al. Subchondral cysts create increased intraosseous stress in early knee OA: A finite element analysis using simulated lesions.[J] .Bone, 2011, 48: 639-46.

17. Osawa Yusuke, Seki Taisuke, Okura Toshiaki et al. Long-term outcomes of curved intertrochanteric varus osteotomy combined with bone impaction grafting for non-traumatic osteonecrosis of the femoral head.[J] .Bone Joint J, 2021, null: 665-671.

18. Deng Wang, Wang Zhaolun, Zhou Yixin et al. Does prior core decompression have detrimental effect on subsequent total hip arthroplasty for osteonecrosis of the femoral head: A systematic review and meta-analysis.[J] .Orthop Traumatol Surg Res, 2020, 106: 1553-1560.

19. Ohzono K, Saito M, Sugano $\mathrm{N}$ et al. The fate of nontraumatic avascular necrosis of the femoral head. A radiologic classification to formulate prognosis.[J] .Clin Orthop Relat Res, 1992, undefined: 73-8. 
20. Kubo Yusuke, Motomura Goro, Ikemura Satoshi et al. The effect of the anterior boundary of necrotic lesion on the occurrence of collapse in osteonecrosis of the femoral head.[J] .Int Orthop, 2018, 42: 1449-1455.

21. Yu Tong,Xie Limin,Chu Fulei, A sclerotic rim provides mechanical support for the femoral head in osteonecrosis.[J] .Orthopedics, 2015, 38: e374-9.

22. Xu Mingjian, Motomura Goro, Ikemura Satoshi et al. Proximal femoral morphology after transtrochanteric posterior rotational osteotomy for osteonecrosis of the femoral head: A threedimensional simulation study.[J]. Orthop Traumatol Surg Res, 2020, 106: 1569-1574.

23. Røhl L, Larsen E, Linde F et al. Tensile and compressive properties of cancellous bone.[J] .J Biomech, 1991, 24: 1143-9.

24. Shi Shan, Luo Ping, Sun Li et al. Prediction of the progression of femoral head collapse in ARCO stage 2-3A osteonecrosis based on the initial bone resorption lesion.[J] .Br J Radiol, 2021, 94: 20200981.

25. McCollum D E, Mathews R S,O'NEIL M T, Aseptic necrosis of the femoral head: associated diseases and evaluation of treatment.[J].South Med J, 1970, 63: 241-53.

26. Zhu Wanbo, Zhang Xianzuo, Fang Shiyuan et al. Deep Learning Improves Osteonecrosis Prediction of Femoral Head After Internal Fixation Using Hybrid Patient and Radiograph Variables.[J] .Front Med (Lausanne), 2020, 7: 573522.

27. Li Qianchun, Chen Rigao, Yu Yang et al. Extracorporeal shockwave therapy combined with multiple drilling and intramedullary drug injection for treating early-stage Femur Head Necrosis: Protocol for a randomized controlled trial.[J].Medicine (Baltimore), 2020, 99: e22598.

28. Yan Zijian,Zhan Jingdi,Qi Weihui et al. The Protective Effect of Luteolin in Glucocorticoid-Induced Osteonecrosis of the Femoral Head.[J] .Front Pharmacol, 2020, 11: 1195.

\section{Figures}




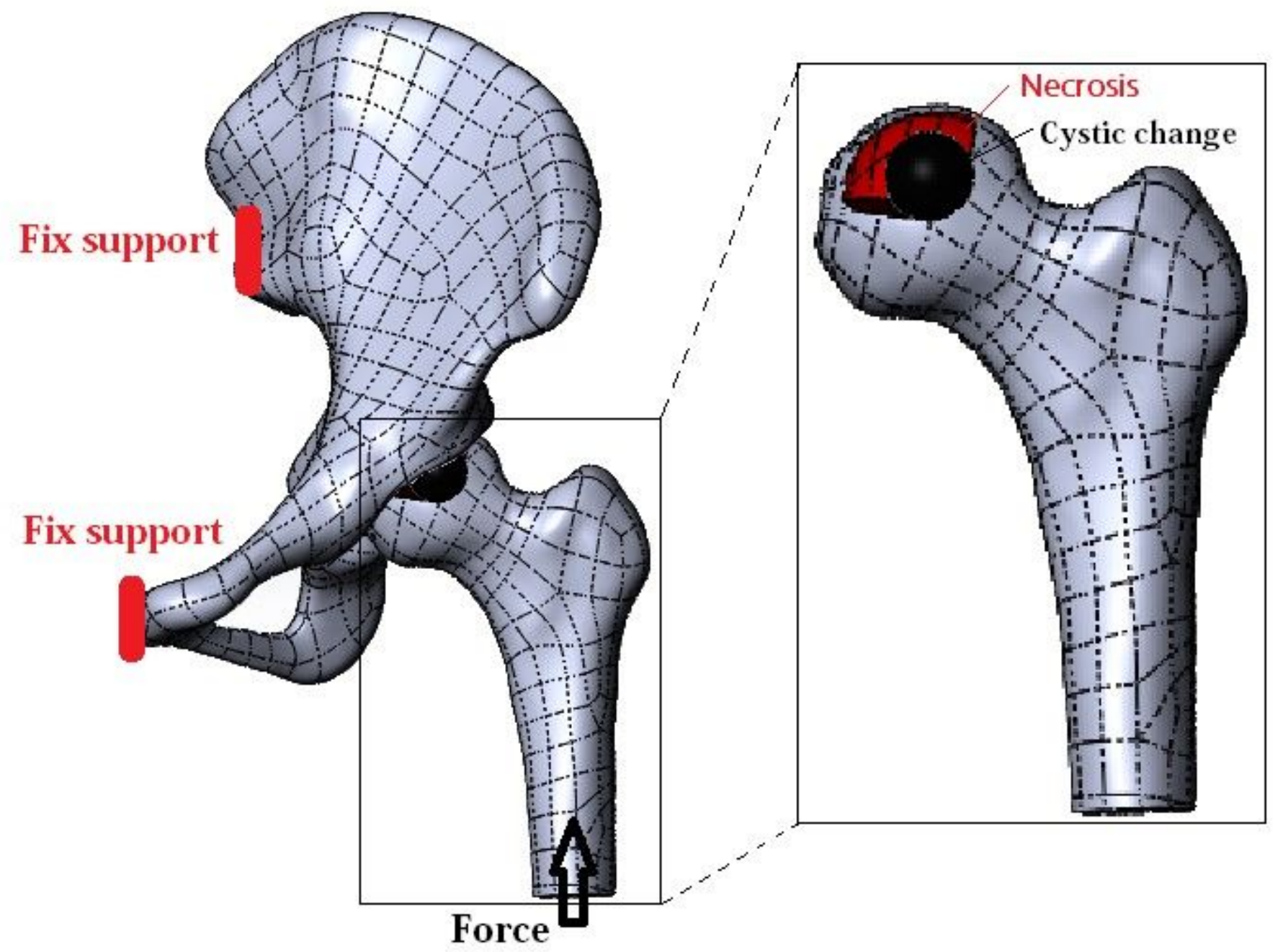

Figure 1

ONFH hip joint model with cystic change area and the diagram of boundary conditions and loading force settings 


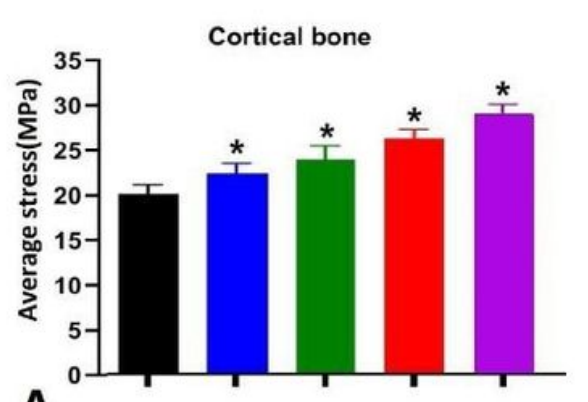

A

Cortical bone

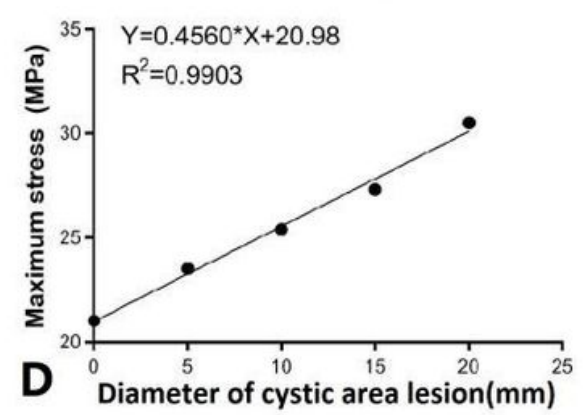

Necrotic zone

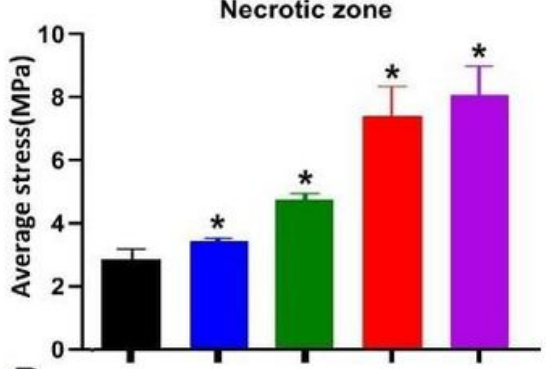

Necrotic zone

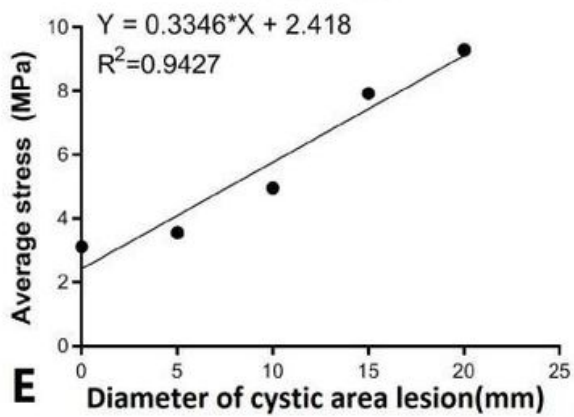

Around cystic area

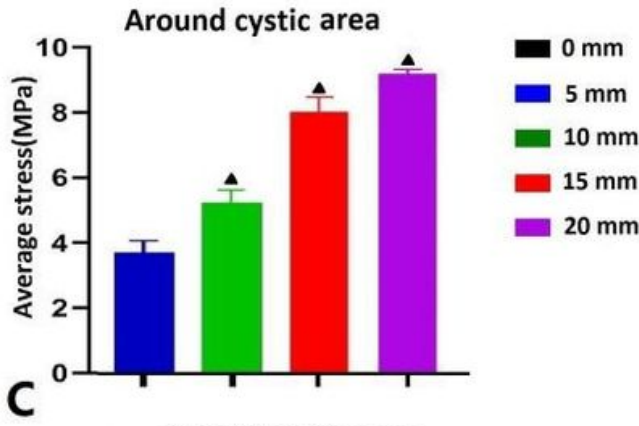

Around cystic area

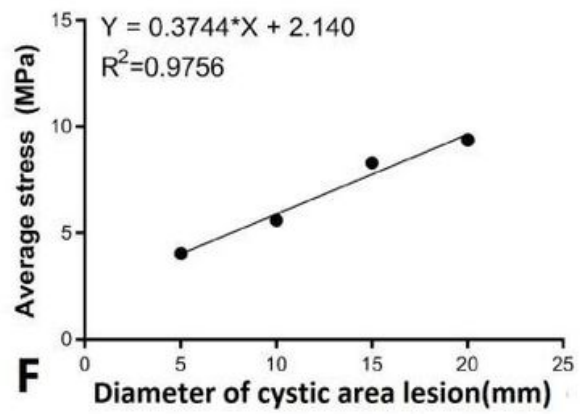

Figure 2

The average stress of each model and relationship between maximum stress and cystic area diameter(AC: Different models average stress of cortical bone, necrotic zone and around cystic area. D-F:

Relationship between the cortical bone, necrotic zone and around cystic area maximum von Mises stress of model and cystic area diameter.) *: Compared with the $0 \mathrm{~mm}$ model, the difference was statistically significant. $\mathbf{\Delta}$ :Compared with the $5 \mathrm{~mm}$ model, the difference was statistically significant. 


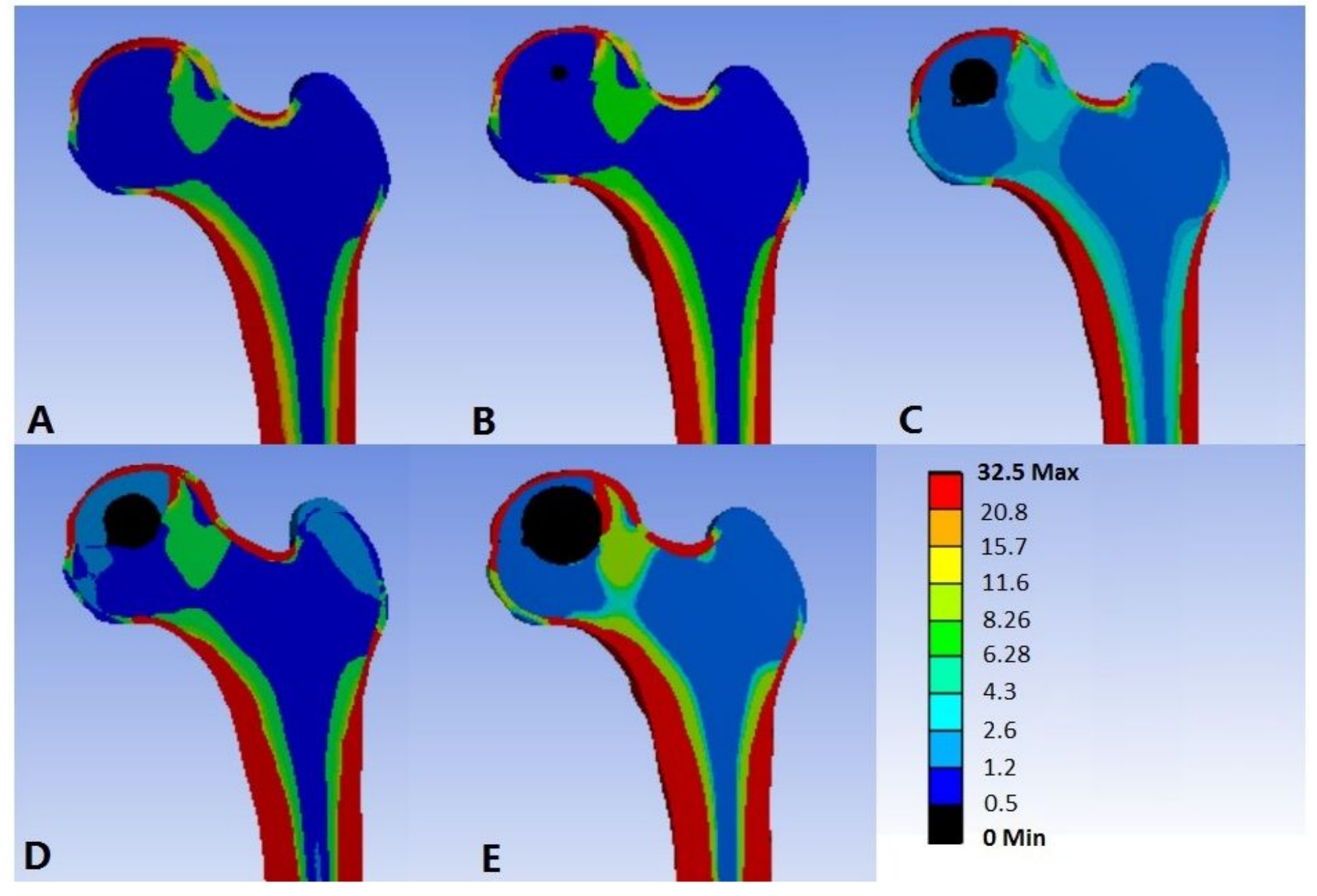

Figure 3

Finite element stress cloud diagrams of different models of femur.(A: Diameter of cystic change area $0 \mathrm{~mm}, \mathrm{~B}$ :Diameter of cystic change area $5 \mathrm{~mm}, \mathrm{C}$ :Diameter of cystic change area $10 \mathrm{~mm}, \mathrm{D}$ :Diameter of cystic change area $15 \mathrm{~mm}$, E:Diameter of cystic change area $20 \mathrm{~mm}$.) 

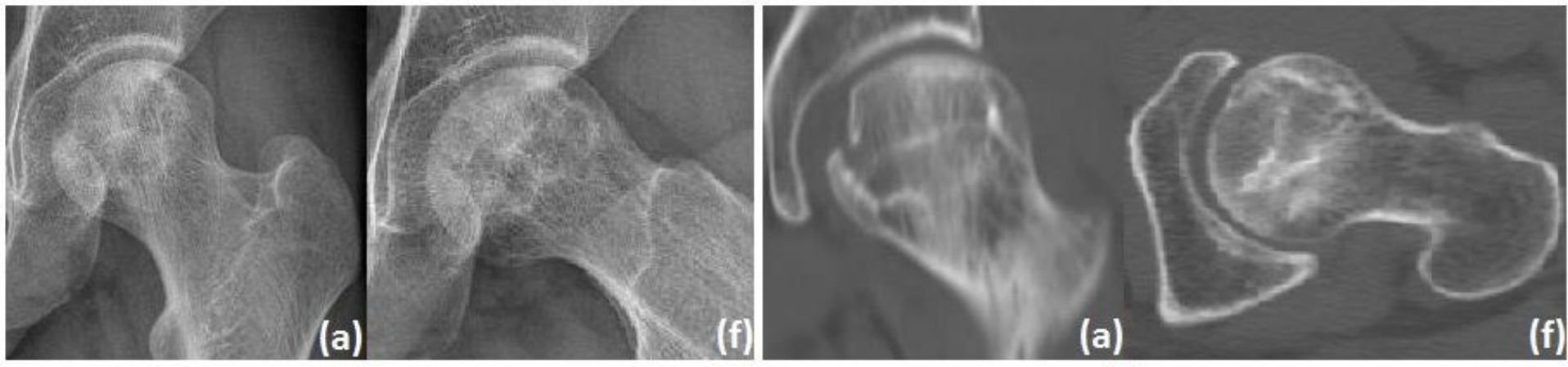

\section{A}

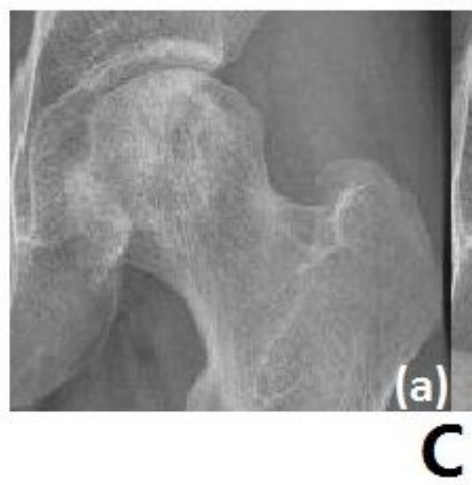

B

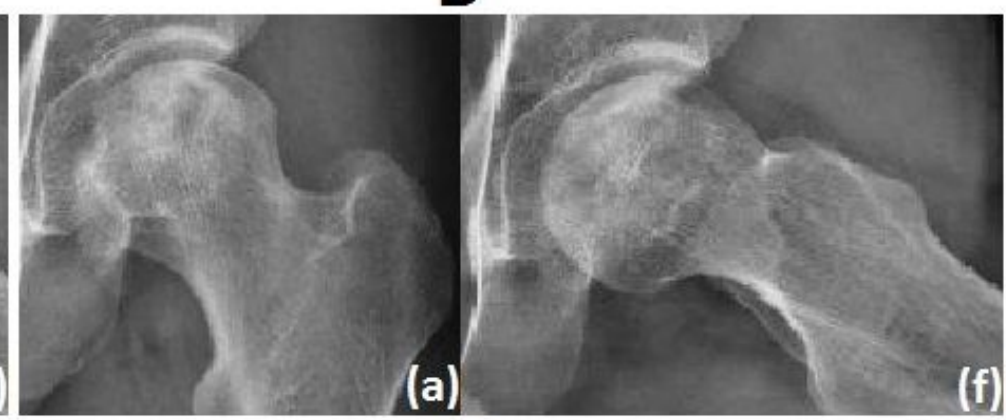

D

Figure 4

Typical case of ONFH without cystic area.(A: Radiograph imaging at an anteroposterior (a) and frog (f) lateral view indicated extensive necrosis of the left femoral head. B: CT showed no obvious cystic change area. C: 6 months follow-up. D: 1.5 years follow-up.)

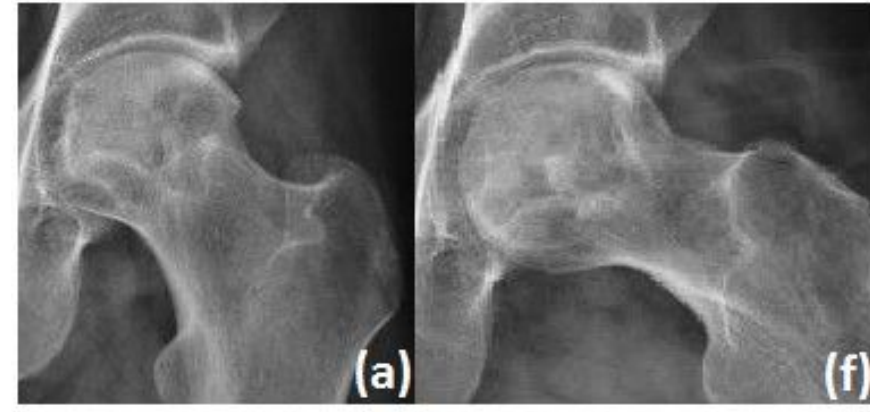

A

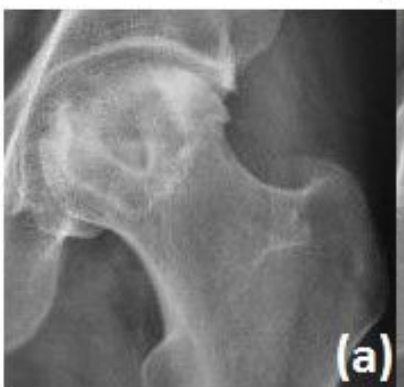

(a)

C (f)
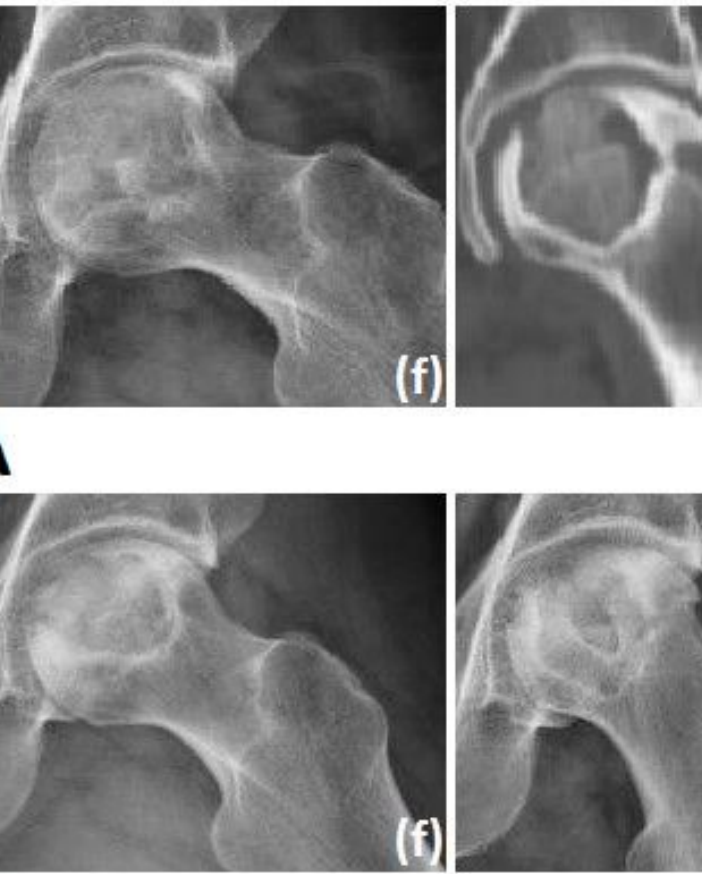

(f)

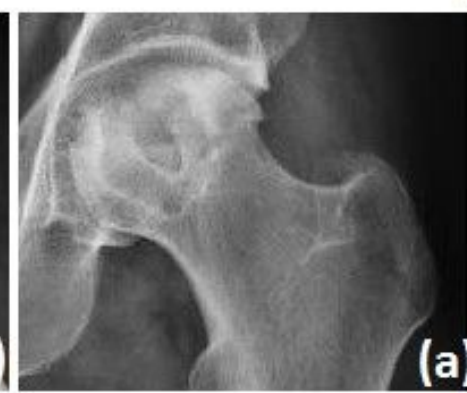

(a)

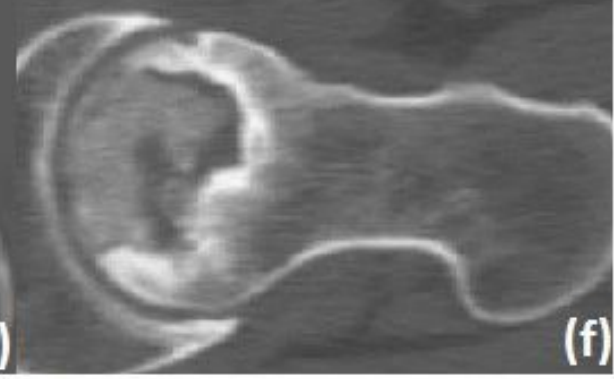

B

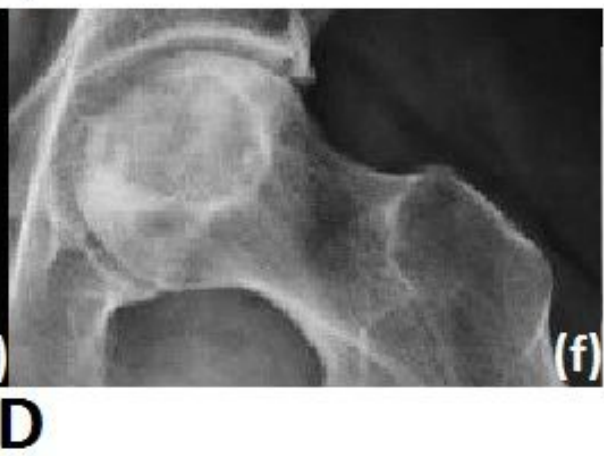

Figure 5 
Typical case of ONFH with cystic area.(A: Radiograph imaging at an anteroposterior (a) and frog (f) lateral view showed large-scale necrosis of the left femoral head. B: CT showed obvious cystic area. C: 1 year follow-up. D: 2 years follow-up.) 